\title{
Antacid use and subclinical interstitial lung disease: the MESA study
}

To the Editor:

Idiopathic pulmonary fibrosis (IPF), a prevalent fibrotic idiopathic interstitial lung disease (ILD), is thought to result from recurrent subclinical alveolar epithelial cell (AEC) injury followed by abnormal repair and fibrogenesis [1]. Gastro-oesophageal reflux disease (GORD) has been implicated in AEC injury in IPF [2]. GORD is a prevalent comorbidity in adults with IPF [3], and the gastric enzyme pepsin has been identified in bronchoalveolar lavage fluid of patients with acute IPF exacerbations [4], suggesting a role of micro-aspiration in disease progression.

Nevertheless, the causal relationship between GORD and IPF remains controversial and unproven. If GORD is a cause of IPF, it should precede the onset of fibrosis, yet most studies examine clinically apparent (and therefore advanced) disease, long after AEC injury and early extracellular matrix (ECM) remodelling have evolved into fibrosis.

We and others have begun investigating early, subclinical ILD, to identify risk factors that precede clinically evident ILD. Two computed tomography (CT)-based subclinical ILD phenotypes have been validated $[5,6]$ in population-based studies: high attenuation areas (HAAs, the percentage of lung voxels having CT attenuation values between -600 and -250 Hounsfield units (HU)) and interstitial lung abnormalities. We hypothesised that antacid use would be associated with lower HAAs in community-dwelling adults.

The Multi-Ethnic Study of Atherosclerosis (MESA) is a National Heart, Lung, and Blood Institute-funded prospective cohort study of 6814 adults aged 45-84 years sampled from six communities across the USA who underwent cardiac CT imaging between 2000 and 2002. These scans include sufficient lung acquisition to allow evaluation for subclinical ILD [6]. MESA enrolment, CT protocols, HAA measurement and other variables have been described previously [6,7]. Study participants brought their medication bottles to the study visit. Trained staff recorded the names of each medication. MESA was approved by the institutional review boards at all centres and all participants provided informed consent.

We used multivariable linear regression to examine the association between antacid use and natural log-transformed percent HAA on baseline cardiac CT scans $(n=6812)$. Analyses were adjusted for age, gender, race, education level, site, body mass index, height, waist circumference, total volume of imaged lung, total volume of emphysema, radiation dose, estimated glomerular filtration rate, smoking status and pack-years of smoking. To address possible confounding by indication, we adjusted for use of alcohol, non-steroidal anti-inflammatory medications (NSAID), cyclooxygenase-2 inhibitors (COX2), oral corticosteroids and aspirin. We performed analyses stratified on smoking status and the use of NSAIDs, COX2 inhibitors and oral corticosteroids.

The characteristics of MESA participants have been published [6]. The mean \pm SD age of the HAA cohort was $62.2 \pm 10.2$ years, $47 \%$ were men, $13 \%$ were current smokers and $37 \%$ were former smokers. At baseline, 422 (6.2\%) subjects reported using proton pump inhibitors (PPIs) and 328 (4.8\%) reported using over-the-counter or prescription histamine-2-receptor blockers $(\mathrm{H} 2 \mathrm{~B}) .18$ subjects $(0.2 \%)$ reported using both PPIs and H2Bs. The mean \pm SD HAA is $5.1 \pm 3.1 \%$, and the median is $4.3 \%$ (range $1.2-48.9 \%$ ).

In unadjusted analyses, baseline PPI use was associated with lower HAA, suggesting a reduction in subclinical ILD (mean percent decrement in HAA among PPI users 6.7\%, 95\% CI 3.9-9.3\%; p<0.001). In adjusted analyses, PPI use was associated with a 3.8\% decrement in HAA (95\% CI 1.6-6.0\%; p<0.001) (figure 1). This association was similar among ever smokers and never smokers ( $p$-value for interaction

@ERSpublications

Proton pump inhibitor use is associated with a reduction in subclinical interstitial lung disease http://ow.ly/dbFN30aoRPf

Cite this article as: Restivo MD, Podolanczuk A, Kawut SM, et al. Antacid use and subclinical interstitial lung disease: the MESA study. Eur Respir J 2017; 49: 1602566 [https://doi.org/10.1183/13993003.025662016]. 


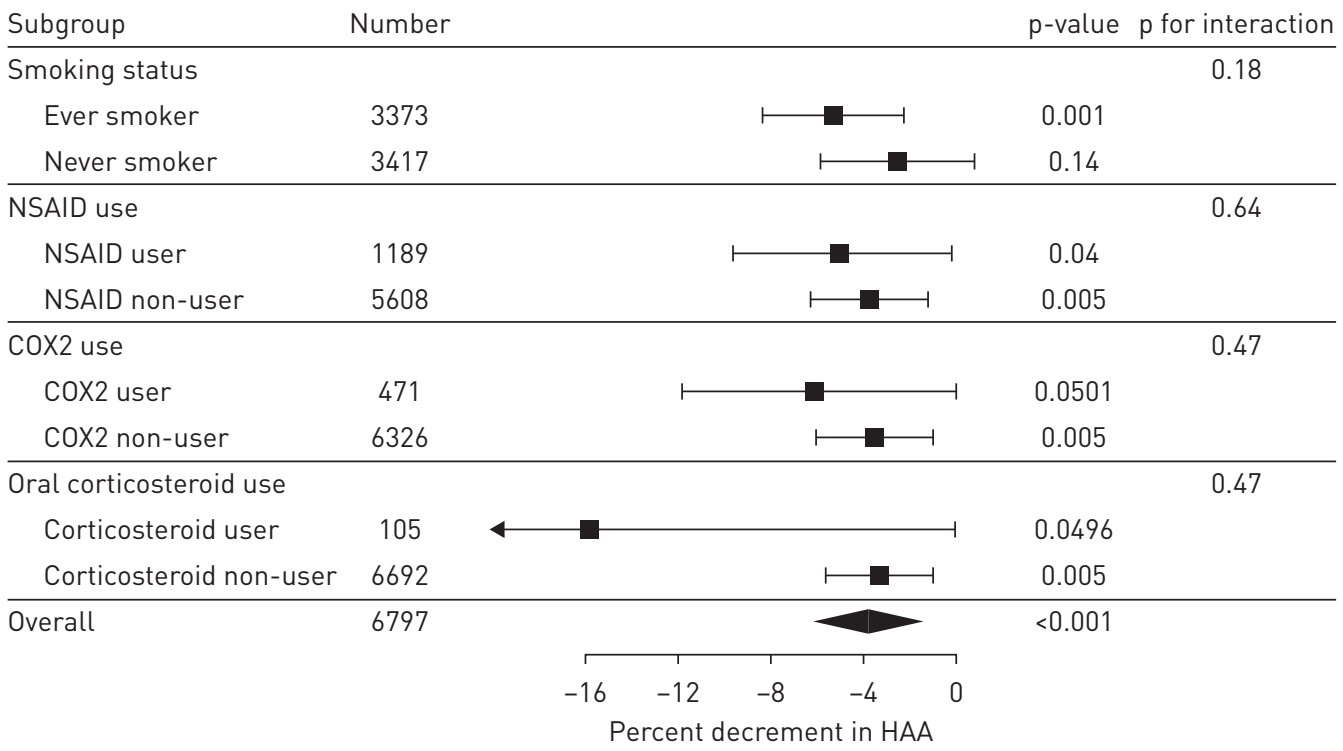

FIGURE 1 Forest plot for fully adjusted stratified analyses of percent decrement in high attenuation areas (HAAs). NSAID: non-steroidal anti-inflammatory medication; COX2: cyclooxygenase-2 inhibitors.

between ever smoking and PPI use was 0.18), although the association among never smokers was non-significant: PPI use was associated with an adjusted mean HAA decrement of 5.3\% (95\% CI 2.2-8.3\%; $\mathrm{p}=0.001$ ) among ever smokers and 2.5\% (95\% CI -0.8-5.7\%; $\mathrm{p}=0.14$ ) among never smokers. The association between PPI use and HAA varied by corticosteroid use ( $\mathrm{p}$-value for interaction between corticosteroid and PPI use was 0.007). PPI use was associated with an adjusted mean HAA decrement of 15.8\% (95\% CI 0.01-29.1\%; $\mathrm{p}=0.0496)$ among corticosteroid users $(\mathrm{n}=105)$ and $3.4 \%$ (95\% CI 1.0-5.6\%; $\mathrm{p}=0.005$ ) among non-users. The association between PPI use and HAA was similar between NSAID users and non-users and between COX2 users and non-users (figure 1). There was no significant association between H2B use and HAA in fully adjusted analyses $(-1.2 \%, 95 \% \mathrm{CI}-3.7-1.4 \%$; $\mathrm{p}=0.36)$.

We are the first to show that PPI use, but not $\mathrm{H} 2 \mathrm{~B}$ use, is associated with a reduction in the percentage of areas of increased CT lung attenuation in community-dwelling adults sampled without regard to respiratory or gastrointestinal symptoms or disease and independent of the use of NSAIDs, COX2 inhibitors, aspirin or oral corticosteroids. Although the association may be stronger among smokers, there was little statistical evidence that the association varied by smoking status. The association may vary by oral corticosteroid use although, given the small number of subjects using corticosteroids, this association should be interpreted cautiously. To our knowledge, there are neither prior studies evaluating PPI therapy and subclinical ILD nor clinical trials of PPI to prevent ILD. In observational studies of IPF, antacid therapy is associated with slower decline in lung function [8,9] and reduced mortality [8]. However, antacid therapy in IPF has also been associated with increased risk of infection [10]. Based on these limited data, current American Thoracic Society/European Respiratory Society/Japanese Respiratory Society/Latin American Thoracic Association guidelines for IPF management conditionally recommend antacid use to treat IPF [11].

Chronic micro-aspiration has been implicated as a cause of AEC injury leading to IPF in susceptible individuals. Our novel observation that PPI use was associated with less HAA, a measure of subclinical injury and inflammation, might be explained by attenuation of AEC injury due to the reduced acidity of gastric refluxate. Prior studies have implicated gastric acid in the increased production of transforming growth factor- $\beta$ (TGF- $\beta$ ) by AECs and increased fibroblast proliferation [12]. Our findings may also be explained by PPI-mediated increases in detoxifying anti-inflammatory enzymes, such as glutathione S-transferase and anti-oxidant stress proteins, increased transcription of the anti-inflammatory and anti-proliferative enzyme haem-oxygenase- 1 and inhibition of TGF- $\beta$-mediated collagen production by fibroblasts [13-15]. PPIs also mitigate lung inflammation and fibrosis in a bleomycin rat model [15].

There are several limitations to our study. First, MESA did not capture clinical diagnoses, such as GORD. It is possible that antacid use is a marker of GORD, and our observations are attributable to the association between GORD and ILD. If true, our data would support the hypothesis that GORD is associated with less, not more, HAA, an association that does not seem plausible given previous work [2-4], and is inconsistent with the absence of an association between H2Bs and HAA. Second, our observational study is subject to residual and unmeasured confounders. MESA took careful measurement of potential confounders and we 
have controlled for these in our analyses. Third, our analyses are cross-sectional in nature, and the duration of medication use prior to imaging is unknown. Fourth, the histopathology of HAA is unknown and, given the possible interaction with smoking status, may reflect a spectrum of pathological changes including smoking-related changes, rather than a single pathologic entity.

In summary, the use of PPIs was associated with fewer HAAs on CT imaging of a large sample of asymptomatic community-dwelling middle aged and older adults. Our data suggest that investigators may wish to consider further investigation of the role of PPI therapy to attenuate early AEC injury and incident ILD in at-risk populations.

Michaela D. Restivo $\odot^{1}$, Anna Podolanczuk ${ }^{1}$, Steven M. Kawut ${ }^{2}$, Ganesh Raghu ${ }^{3}$, Peter Leary ${ }^{3}$, R. Graham Barr ${ }^{1,4}$ and David J. Lederer $\oplus^{1,4}$

${ }^{1}$ Dept of Medicine, Columbia University Medical Center, New York, NY, USA. ${ }^{2}$ Dept of Medicine and the Center for Clinical Epidemiology and Biostatistics, Perelman School of Medicine at the University of Pennsylvania, Philadelphia, PA, USA. ${ }^{3}$ Dept of Medicine, University of Washington, Seattle, WA, USA. ${ }^{4}$ Dept of Epidemiology, Columbia University Medical Center, New York, NY, USA.

Correspondence: David J. Lederer, Medicine and Epidemiology, Columbia University Medical Center, 161 Fort Washington Avenue, Room 3-321A, New York, NY 10032, USA. E-mail: davidlederer@columbia.edu

Received: Dec 312016 | Accepted after revision: Feb 112017

Support statement: The work is funded by the National Institutes of Health (contracts HHSN268201500003I, N01-HC-95159, N01-HC-95160, N01-HC-95161，N01-HC-95162，N01-HC-95163，N01-HC-95164，N01-HC-95165, N01-HC-95166, N01-HC-95167, N01-HC-95168 and N01-HC-95169 and grants UL1-TR-000040, UL1-TR-001079, R01-HL-103676, RC1-HL100543, R01-HL-093081, R01-HL-077612, T32-HL-105323 and K24-HL-131937), by the Pulmonary Fibrosis Foundation, and by the Rocco Guinta Research Fund. Funding information for this article has been deposited with the Crossref Funder Registry.

Conflict of interest: Disclosures can be found alongside this article at erj.ersjournals.com

\section{References}

King TE Jr, Pardo A, Selman M. Idiopathic pulmonary fibrosis. Lancet 2011; 378: 1949-1961.

2 Savarino E, Carbone R, Marabotto E, et al. Gastro-oesophageal reflux and gastric aspiration in idiopathic pulmonary fibrosis patients. Eur Respir J 2013; 42: 1322-1331.

3 Raghu G, Freudenberger TD, Yang S, et al. High prevalence of abnormal acid gastro-oesophageal reflux in idiopathic pulmonary fibrosis. Eur Respir J 2006; 27: 136-142.

4 Lee JS, Song JW, Wolters PJ, et al. Bronchoalveolar lavage pepsin in acute exacerbation of idiopathic pulmonary fibrosis. Eur Respir J 2012; 39: 352-358.

5 Washko GR, Hunninghake GM, Fernandez IE, et al. Lung volumes and emphysema in smokers with interstitial lung abnormalities. N Engl J Med 2011; 364: 897-906.

6 Podolanczuk AJ, Oelsner EC, Barr RG, et al. High attenuation areas on chest computed tomography in community-dwelling adults: the MESA study. Eur Respir J 2016; 48: 1442-1452.

7 Bild DE, Bluemke DA, Burke GL, et al. Multi-Ethnic Study of Atherosclerosis: objectives and design. Am J Epidemiol 2002; 156: 871-881.

8 Lee JS, Ryu JH, Elicker BM, et al. Gastroesophageal reflux therapy is associated with longer survival in patients with idiopathic pulmonary fibrosis. Am J Respir Crit Care Med 2011; 184: 1390-1394.

9 Lee JS, Collard HR, Anstrom KJ, et al. Anti-acid treatment and disease progression in idiopathic pulmonary fibrosis: an analysis of data from three randomised controlled trials. Lancet Respir Med 2013; 1: 369-376.

10 Kreuter M, Wuyts W, Renzoni E, et al. Antacid therapy and disease outcomes in idiopathic pulmonary fibrosis: a pooled analysis. Lancet Respir Med 2016; 4: 381-389.

11 Raghu G, Rochwerg B, Zhang Y, et al. An official ATS/ERS/JRS/ALAT clinical practice guideline: treatment of idiopathic pulmonary fibrosis. An update of the 2011 clinical practice guideline. Am J Respir Crit Care Med 2015; 192: e3-19.

12 Perng DW, Chang KT, Su KC, et al. Exposure of airway epithelium to bile acids associated with gastroesophageal reflux symptoms: a relation to transforming growth factor- $\beta 1$ production and fibroblast proliferation. Chest 2007; 132: $1548-1556$.

13 Becker JC, Grosser N, Waltke C, et al. Beyond gastric acid reduction: proton pump inhibitors induce heme oxygenase-1 in gastric and endothelial cells. Biochem Biophys Res Commun 2006; 345: 1014-1021.

14 Naito Y. Anti-inflammatory and anti-oxidative properties of proton pump inhibitors. J Clin Biochem Nutr 2007; 41: 82-83.

15 Ghebremariam YT, Cooke JP, Gerhart W, et al. Pleiotropic effect of the proton pump inhibitor esomeprazole leading to suppression of lung inflammation and fibrosis. J Transl Med 2015; 13: 249. 